OPEN ACCESS

Edited by:

Christos Dimitrakopoulos, University of Massachusetts Amherst, United States

Reviewed by: Mads Brandbyge, Technical University of Denmark, Denmark Shengjun Yuan, Wuhan University, China

*Correspondence: Ke-Qiu Chen keqiuchen@hnu.edu.cn

Specialty section: This article was submitted to Condensed Matter Physics,

a section of the journal

Frontiers in Physics

Received: 27 November 2021 Accepted: 14 February 2022

Published: 04 March 2022

Citation:

Zeng Y-J, Liu Y-Y, Pan H, Ding Z-K, Zhou $W-X$, Tang $L-M, L i B$ and Chen K-Q (2022) Thermoelectric Conversion From Interface

Thermophoresis and

Piezoelectric Effects.

Front. Phys. 10:823284.

doi: 10.3389/fphy.2022.823284

\section{Thermoelectric Conversion From Interface Thermophoresis and Piezoelectric Effects}

\author{
Yu-Jia Zeng ${ }^{1}$, Yue-Yang Liu ${ }^{2}$, Hui Pan ${ }^{1}$, Zhong-Ke Ding ${ }^{1}$, Wu-Xing Zhou ${ }^{3}$, Li-Ming Tang ${ }^{1}$, \\ Baowen $\mathrm{Li}^{4}$ and Ke-Qiu Chen ${ }^{1 *}$ \\ ${ }^{1}$ Department of Applied Physics, School of Physics and Electronics, Hunan University, Changsha, China, ${ }^{2}$ Insitute of \\ Semiconductors, Chinese Academy of Sciences, Beijing, China, ${ }^{3}$ School of Materials Science and Engineering, Hunan University \\ of Science and Technology, Xiangtan, China, ${ }^{4}$ Department of Mechanical Engineering, University of Colorado, Boulder, CO, \\ United States
}

Piezoelectric effect has proved itself to be a promising energy conversion mechanism that can convert mechanical energy into electricity. Here, we propose an indirect thermoelectric conversion mechanism based on a combination of the thermophoresis and piezoelectric effects. We first analyze this thermally driven mechanism using a simplified theoretical model and then numerically analyze a molecular dynamics (MD) simulation of a hybrid system constructed of a single-layer $\mathrm{MoS}_{2}$ nanoribbon and a concentric carbon nanotube. We show that the thermophoresis-induced piezoelectric output voltage can reach $3.5 \mathrm{~V}$, and this value can be tuned using a temperature difference. The output voltage obtained using this mechanism is significantly higher than that obtained by heating piezoelectric materials directly. Given the generality of the thermophoresis effect in Van der Waals structures, this mechanism has potential applications in the conversion of thermal energy into electrical energy at the nanoscale level.

Keywords: thermoelectric, thermophoresis, piezoelectric, phonon, carbon nanotube, nanoribbon

\section{INTRODUCTION}

The thermoelectric and piezoelectric effects, which, respectively, convert heat and mechanical energy into electricity, have attracted tremendous attention in the past few decades. Significant efforts have been devoted to improving thermoelectric conversion efficiency by suppressing lattice thermal conductivity [1-8] or engineering electronic band structures [9-17] to enhance the power factor. However, thermoelectric conversion efficiency is still limited by the complex coupling relations between phonons and electrons [18-20]. The piezoelectric effect converts mechanical energy into electricity directly and has been applied in nanowires [21-23], thin films [24], and organic-inorganic perovskites [25]. However, the piezoelectric effect generally converts artificial or ambient mechanical energy rather than heat energy into electrical power. Although piezoelectric materials can also realize thermoelectric conversion via the pyroelectric effect, this relies on a constantly changing temperature [26]. There is still a challenge when it comes to converting heat energy into electrical energy through the piezoelectric effect when the system is at a constant temperature difference. Encouragingly, the experimental observation of the piezoelectric effect in $2 \mathrm{D}$ materials suggests an opportunity for thermoelectric conversion based on piezoelectric materials [27].

Recently, thermally driven piezoelectric conversion has attracted extensive attention in energy conversion [28]. Our previous study has demonstrated that output voltages as large as $0.34 \mathrm{~V}$ can be obtained by thermally induced deflection of GaN nanowires [29]. A similar conversion mechanism 


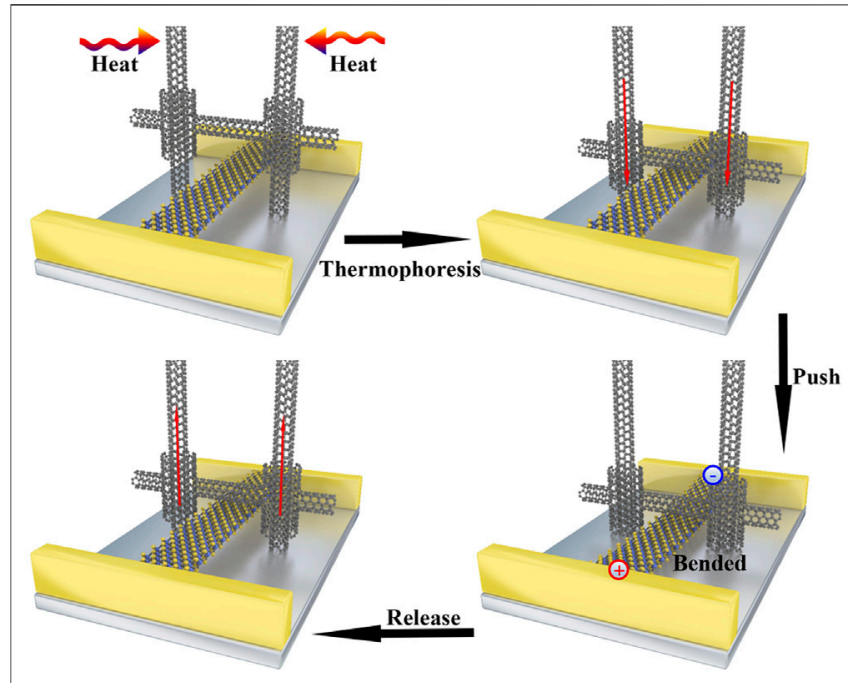

FIGURE 1 | Sketch of the thermoelectric conversion process driven by thermophoresis and piezoelectric effects. The concentric carbon nanotube is made of an [4, 4] inner tube and [8, 8] outer tube with the length of 36.9 and $3.3 \mathrm{~nm}$, respectively. An armchair edge $\mathrm{MoS}_{2}$ nanoribbon with the length of $21.9 \mathrm{~nm}$ is adopted, and the different widths of the nanoribbon (1.58, 2.21, and $2.84 \mathrm{~nm}$ ) are investigated for comparison.

has also been observed in $2 \mathrm{D}$ materials such as $\mathrm{h}-\mathrm{BN}$ nanoribbons [30]. This conversion mechanism depends on direct heating to trigger the deflection of piezoelectric materials. Another promising mechanism called the thermophoresis effect, which converts thermal energy into mechanical energy, has also been confirmed by theory [31,32] and experiment [33] and has shown great potential for application in energy conversion [34]. According to the motion style, thermophoretic motion can be classified into two major categories. One is linear motion, in which the mover undergoes translational motion relative to the stator. The other one is known as rotation, in which the rotor rotates along the axis of the stator. The thermophoresis effect has been identified in many different materials that are connected by Van der Waals (vdW) interactions, such as bilayer graphene [35], h-BN/graphene vdW heterostructures [36], and concentric double-walled carbon nanotubes (CNTs) [37-40].

Here, we theoretically propose a thermoelectric conversion mechanism based on a combination of interface thermophoresis and piezoelectric effects. This allows for energy conversion from heat to mechanical to electrical energy, by combining the thermophoresis and piezoelectric effects into one system. The key point here is the generation of a considerable low-frequency periodic strain in the piezoelectric material via a thermophoresis oscillator (mover CNT). Generally, large vibrational amplitude can be effectively induced through a resonance effect, with the frequency of an external excitation (such as the temperature gradient and/or external force) being equal to or close to the natural (eigen) frequency of the material [41]. However, the vibrations are composed of many different high-frequency lattice waves when a temperature difference is directly applied to piezoelectric materials [42]. Such high-frequency vibrations are hardly harvested by piezoelectric materials.
Since the mover and stator of the thermophoretic system are bonded by vdW interactions, the dynamical properties of the thermophoretic mover are dependent not only on the thermal properties of the stator but also on the strength of the vdW interactions between the stator and mover. Recently, theoretical studies have shown that the vibration induced by the thermophoresis effect can be periodic and tuned by a temperature difference [38, 40, 43]. Therefore, the thermophoresis effect is a promising way to realize thermally induced piezoelectric conversion. Based on the thermophoresis effect, we have designed a composite structure made of a concentric CNT network and a suspended $\mathrm{MoS}_{2}$ nanoribbon with two fixed leads, as depicted in Figure 1. The mover CNT (outer tube) will be pushed to move when a temperature difference is established between both ends of the stator CNT (inner tube). The mover CNT then serves as the strain source for the $2 \mathrm{D}$ piezoelectric material to realize the thermally driven piezoelectric conversion.

\section{RESULTS AND DISCUSSION}

The entire piezoelectric vibration system can be simplified into an oscillator model with an external driving force. We first present an atomic chain model with two fixed leads to illustrate the dynamical properties of the system, shown in Figure 2A. Considering the mover CNT mainly acts on the central region of the nanoribbon, a similar periodic driving force is applied to the central area of the atomic chain to serve as the nonequilibrium phonon-transport-induced thermal driving force. Considering the wave nature of the lattice vibrations, the assumption of a periodic thermal driving force is reasonable. We also provide a phonon wave-packet simulation [44] for the phonon-transport-induced thermal driving force to illustrate our assumption (see Supplementary Material). Here, we consider only the nearest neighbor atomic interactions and the internal damping force acting on the oscillator. Then, the equation of motion of the oscillators can be written as follows:

$$
m \ddot{r}+\lambda \dot{r}+k r-\sum_{i} F_{i} \cos \omega_{i} t=0,
$$

where $k$ is the spring constant of the harmonic oscillator, $r$ is the position of the atoms, $m$ is the atomic mass, and $\lambda$ is the damping factor $(\lambda=0.1)$. The external driving forces are a linear combination of forces with different frequencies $\left(\omega_{i}\right)$ and amplitudes $\left(F_{i}\right)$.

As we can see in Figure 2B, in the case of forced vibration, the amplitude of the system in all directions is very small until the driving force is applied. Subsequently, the out-of-plane vibration fluctuates significantly but soon converges to a stable state (with an amplitude of around $0.4 \mathrm{~nm}$ ), and the in-plane vibrations (in the $x$ and $y$ directions) are still very small. In contrast, the amplitude of the elastic vibration is around one magnitude less than that of the forced vibration, and the frequency of the elastic vibration is much higher than that of the forced vibration. We further analyze the vibrational properties in the frequency domain with and without the 

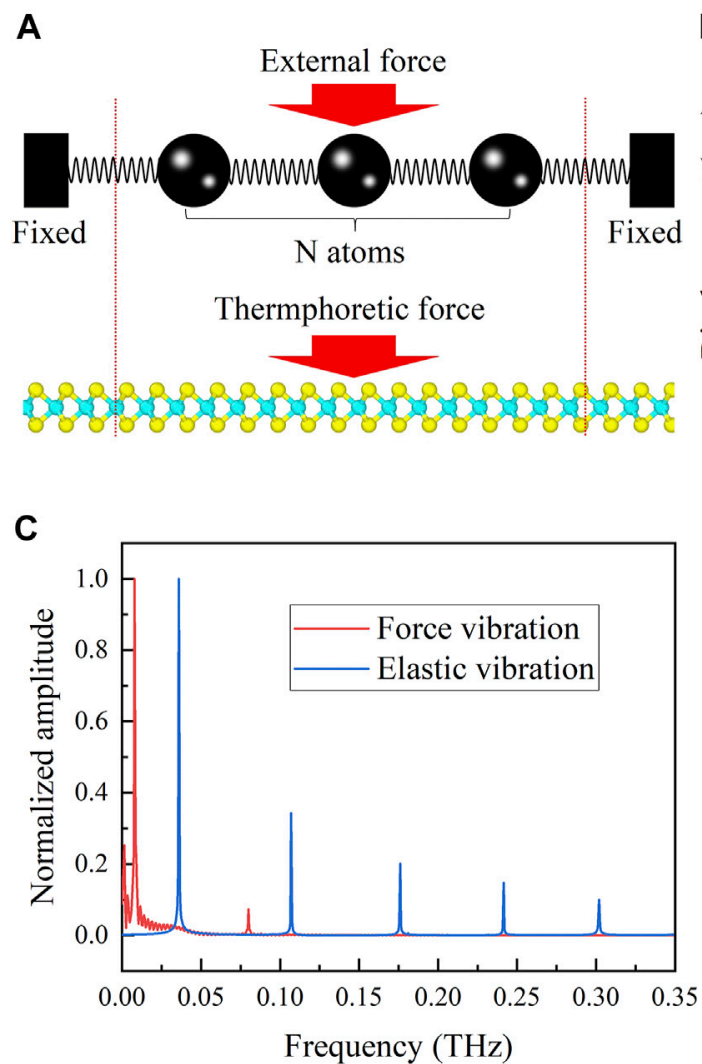
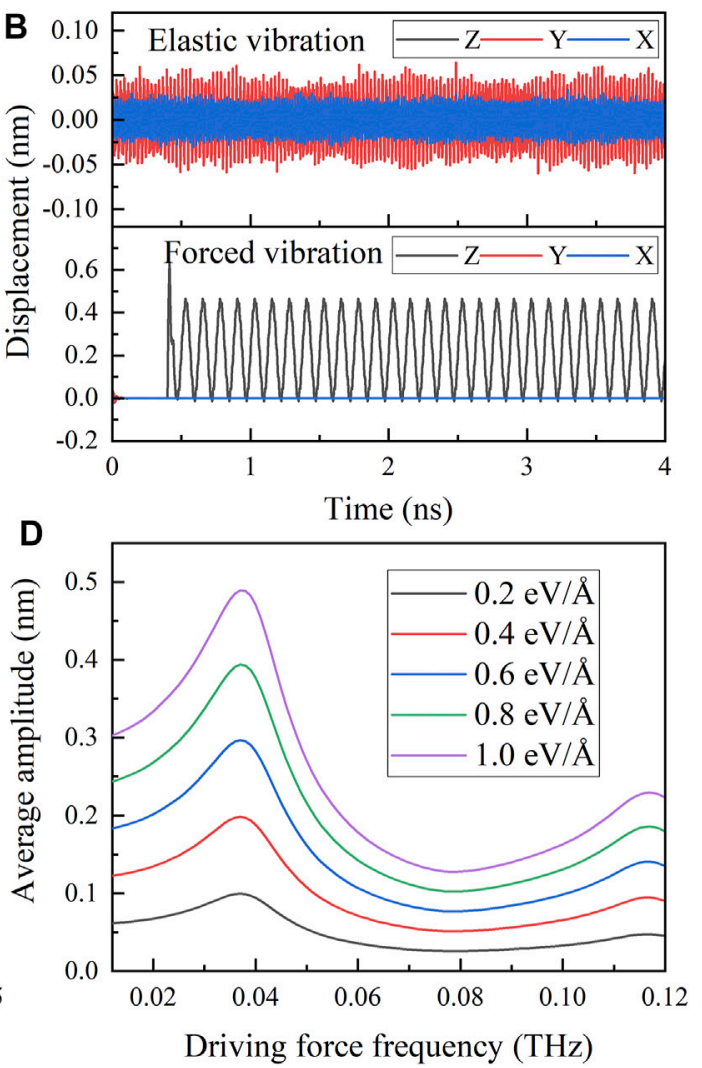

FIGURE 2 | (A) Model of the atomic chain and $\mathrm{MoS}_{2}$ nanoribbon with a periodic thermal driving force. Here, atom number $N=21$. (B) Vibration trajectory of the center atom in the range of 0-4 ns and 10-12 ns (inset), where the driving force amplitude $F_{i}=1,0.5,0.25$ eV/A and the corresponding frequency $\omega_{i}=0.008$, 0.08 , and $0.8 \mathrm{THz}$, respectively. (C) Vibration frequency distribution of forced vibration and elastic vibration (without external driving force and damping force). (D) Vibration amplitude of atomic chain versus the driving force frequency when the driving force amplitude $F_{1}=0.2,0.4,0.6,0.8$, and 1.0 eV/Å, respectively.

external driving force. As we can see in Figure 2C, the natural vibration of the oscillator (without the driving force) displays multi-frequency peaks (blue solid line). By contrast, the vibrational frequency of the oscillator is significantly reduced in the presence of an external driving force (red solid line). There are only two obvious vibration frequencies that can be observed. We further change the driving force frequency to calculate the amplitude of the oscillator (from $0.008 \mathrm{THz}$ to $0.12 \mathrm{THz}$ ). As shown in Figure 2D, when the driving force frequency approaches the natural frequency of the oscillator, the amplitude of the oscillator is significantly enhanced. These results suggest that the vibrational amplitude and frequency of the oscillator can be effectively controlled as long as we can tune the driving force.

The toy model gives positive results for the realization of thermally driven deflection. However, the actual system is far more complex than this toy model. One may wonder whether the thermophoretic driving force is sufficient to induce a considerable low-frequency vibration on the actual piezoelectric material. For this purpose, we further adopt a molecular dynamics (MD) simulation to investigate the thermally driven vibration of the $\mathrm{MoS}_{2}$ nanoribbon in two cases (1): the temperature difference is directly applied at both ends of the suspended $\mathrm{MoS}_{2}$ nanoribbon to trigger the lattice vibrations (2); the temperature difference is applied to both ends of the stator CNT (the inner tube in Figure 1) to induce the thermophoretic motion of the mover CNT (the outer layer CNT in Figure 1), thereby triggering the deflection of the nanoribbon. In both cases, the entire system is first relaxed at the NPT ensemble for $0.2 \mathrm{~ns}$. Next, Nose-Hoover thermostats are applied at both ends of the CNT. The time step is set to $0.1 \mathrm{fs}$. The vibrational trajectory of the $\mathrm{MoS}_{2}$ nanoribbon is collected every 2 ps to characterize the vibration state. The original and modified AIREBO potentials are adopted for the $\mathrm{MoS}_{2}$ and CNT, respectively $[45,46]$. The vdW interactions between the $\mathrm{MoS}_{2}$ and CNT are described by the Lenard-Jones potential with parameters $\sigma_{\mathrm{C}-\mathrm{Mo}}=3.075, \varepsilon_{\mathrm{C}-\mathrm{Mo}}=3.32 \mathrm{meV}$, $\sigma_{\mathrm{C}-\mathrm{S}}=3.513$, and $\varepsilon_{\mathrm{C}-\mathrm{S}}=7.35 \mathrm{meV}$ [47]. All of the MD simulations were performed using the LAMMPS code.

We first discuss the vibrational properties of the $\mathrm{MoS}_{2}$ nanoribbon in the case of direct heating. As shown in Figure 3A, a temperature difference $\Delta \mathrm{T}=210 \mathrm{~K}$ (around $9.6 \mathrm{~K} / \mathrm{nm}$ ) is established at both ends of the suspended nanoribbon to induce lattice vibration. The vibrational trajectory of the $\mathrm{MoS}_{2}$ nanoribbon is shown in Figure 3B, and we can see that the average amplitude is around $0.1 \mathrm{~nm}$ in the out- 

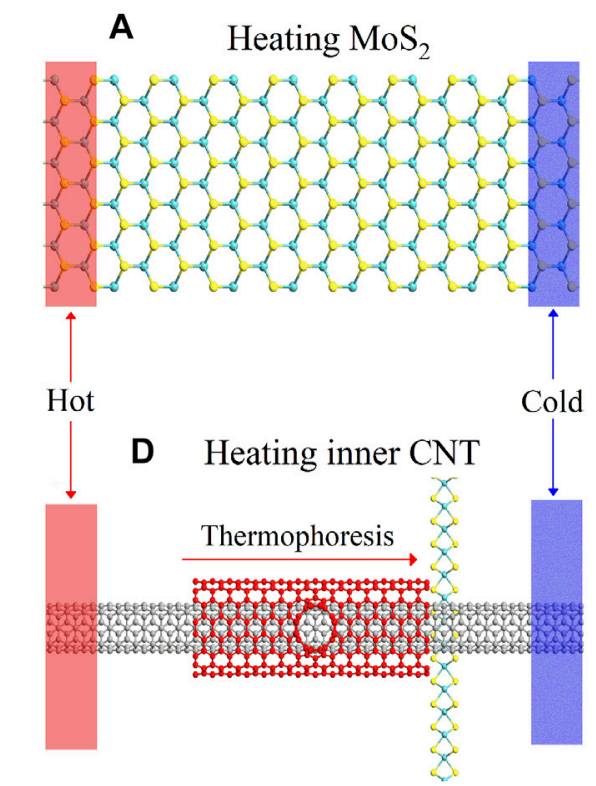
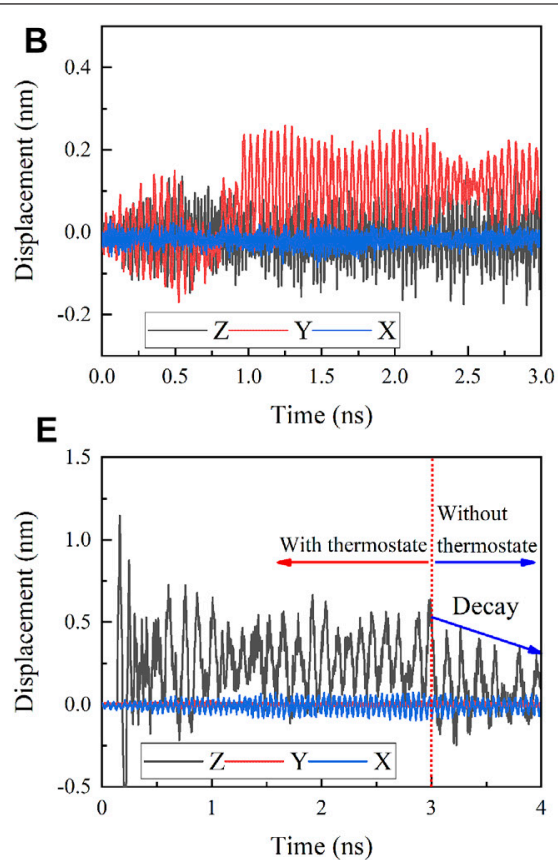

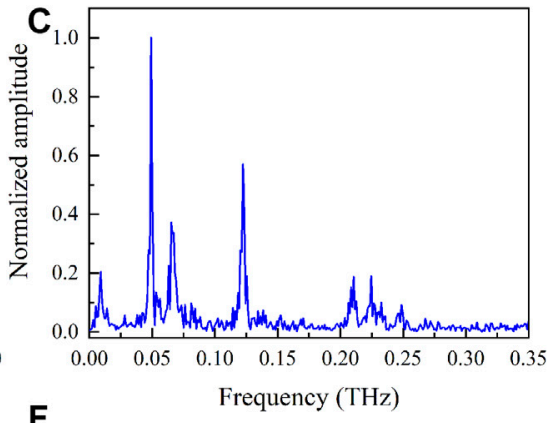

F

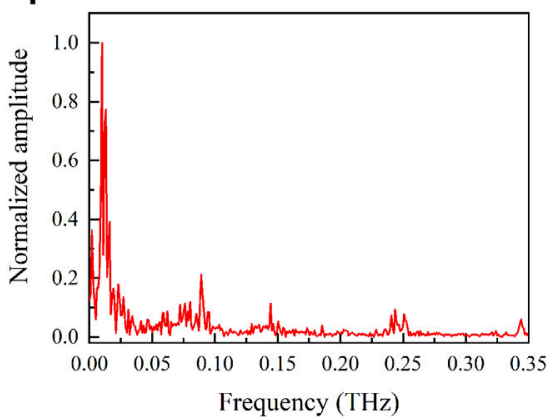

FIGURE 3 | (A) Heated bath at both ends of the $\mathrm{MoS}_{2}$ nanoribbon: (B) the vibration trajectory and (C) the vibration frequency distribution when observed from the center area of the $\mathrm{MoS}_{2}$ nanoribbon. (D) Heated bath on both ends of the inner CNT. (E) Vibration trajectory of the MoS 2 nanoribbon. The left/right side of the red dashed line corresponded to the situation of with and without the heated bath, respectively. (F) Vibration frequency distribution of the MoS $\mathrm{S}_{2}$ nanoribbon when the inner $\mathrm{CNT}$ is heated.

of-plane direction and around $0.2 \mathrm{~nm}$ in the in-plane direction. In addition, as shown in Figure 3C, the frequency domain clearly shows three obvious vibration states at $0.049,0.065$, and $0.122 \mathrm{THz}$. This is in qualitative agreement with the results of the elastic vibration model in theoretical analysis. It is worth noting that the in-plane deflection of the nanoribbon can reach $0.2 \mathrm{~nm}$. This seems to suggest that the direct heating method is a promising way to trigger piezoelectric effects. However, a theoretical study has pointed out that high-frequency vibration reduces the conversion efficiency of piezoelectric materials [48].

As a comparison, we establish the same temperature difference at both ends of the stator CNT (a temperature gradient of around $5.8 \mathrm{~K} / \mathrm{nm}$ ), as shown in Figure 3D. It should be mentioned that this temperature gradient is reasonable. Both theoretical [31, 38] and experimental $[33,49]$ studies suggest that a maximum thermal gradient of around $10 \mathrm{~K} / \mathrm{nm}$ can be imposed on the CNT. The vibrational trajectory of the $\mathrm{MoS}_{2}$ is shown in Figure 3E. We can see that the amplitude of the out-of-plane vibration is around $0.5 \mathrm{~nm}$, while the amplitude of the in-plane vibration is almost negligible. The out-of-plane vibrational amplitude is significantly enhanced, and the in-plane vibrational amplitude is suppressed in comparison with direct heating of the nanoribbon. More importantly, the vibration of the nanoribbon also exhibits good periodicity. This is equivalent to applying a periodic strain in the out-of-plane direction of the nanoribbon. This result is consistent with the simplified model and satisfactorily proves the feasibility of our core idea.

To be prudent, the presence of damping forces in this system should be further verified to exclude false periodicity caused by elastic vibration. As shown on the right side of Figure 3E, we remove the temperature difference after $3 \mathrm{~ns}$, and thus, the whole system is at room temperature $(300 \mathrm{~K})$. The vibration of the $\mathrm{MoS}_{2}$ nanoribbon is damped without external energy input. This proves the reliability of the thermally induced periodic vibration. On another hand, it also suggests that the switching state of the system can be flexibly controlled by applying/removing the temperature difference. More importantly, as shown in Figure 3F, the frequency domain shows a major vibration peak at about $0.01 \mathrm{THz}$. The vibrational frequency of out-of-plane modes is suppressed by up to 5 times compared to the direct heating method, and the amplitude is increased by up to 2 times. For piezoelectric materials, a lower vibrational frequency usually means a higher energy conversion efficiency [48]. This means that the hybrid system driven by the thermophoresis effect will obtain a higher conversion efficiency and output voltage.

Another question is whether the thermophoretic driving force can be effectively regulated in such a system. Recent studies have shown that the natural frequency of materials will be shifted when the ambient temperature is changed [50-53]. This suggests that the frequency of the thermophoretic force can be tuned by changing the temperature difference between the left and right leads. For this reason, we further investigate the temperature-dependent average amplitude of the $\mathrm{MoS}_{2}$ nanoribbon. As shown in Figure 4A, the average amplitude of the $\mathrm{MoS}_{2}$ nanoribbon is increased with increasing temperature difference when $\Delta \mathrm{T}<270 \mathrm{~K}$. There is a maximum amplitude at 

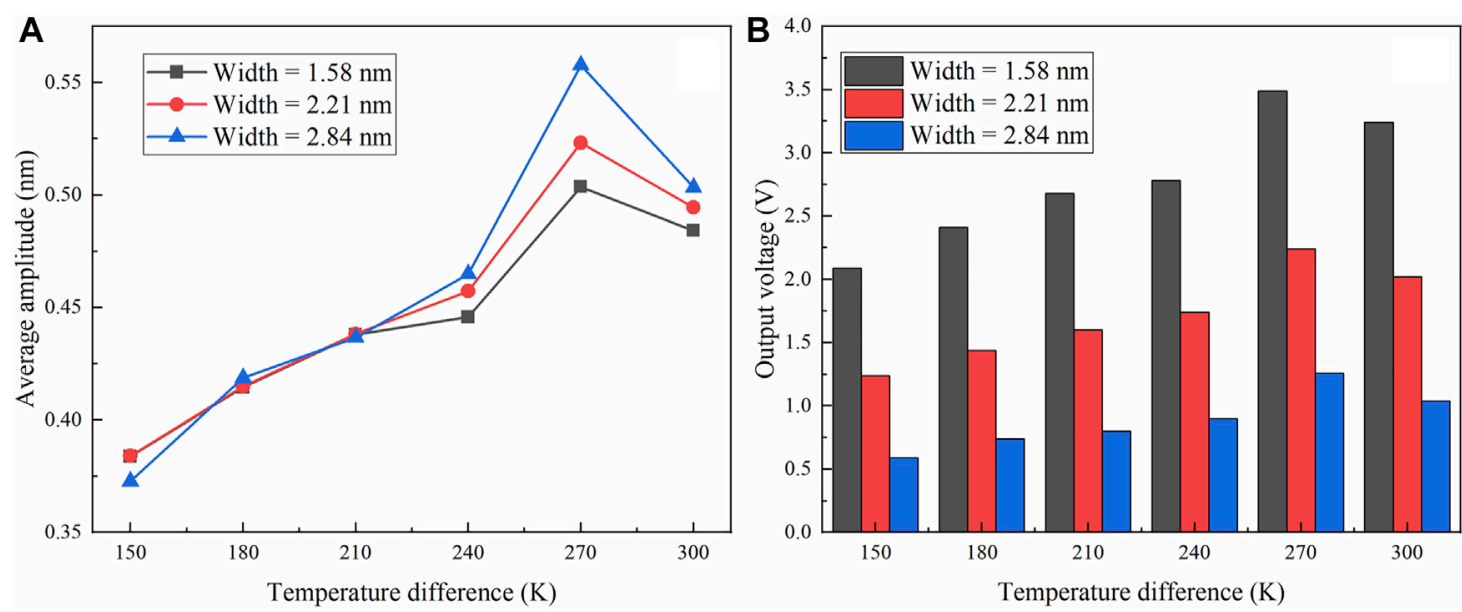

FIGURE 4 | (A) Average amplitude and (B) open-circuit voltage of the $\mathrm{MoS}_{2}$ nanoribbon with different widths as a function of temperature difference.

TABLE 1 | Piezoelectric and elastic constant of $\mathrm{MoS}_{2}$ when normalized by a layer thickness of $0.7 \mathrm{~nm}$.

\begin{tabular}{lcc} 
Width $\mathbf{( n m})$ & Piezoelectric constant $\left(\mathbf{C} / \mathbf{m}^{\mathbf{2}}\right)$ & Elastic constant (GPa) \\
\hline 1.58 & 6.67 & 24.94 \\
2.21 & 3.94 & 29.28 \\
2.84 & 1.91 & 32.31 \\
Bulk & 0.56 & 57.58 \\
\hline
\end{tabular}

$\Delta \mathrm{T}=270 \mathrm{~K}$, which is qualitatively consistent with the amplitude increase as predicted by the theoretical analysis. This effect illustrates that the temperature difference can effectively regulate the driving force frequency and then tune the thermally induced vibrational amplitude.

To confirm the piezoelectric performance, the size-dependent piezoelectric constant of the $\mathrm{MoS}_{2}$ is calculated using a density functional theory (DFT) calculation, as shown in Table 1. The piezoelectric potential distribution under a periodic strain is calculated with the help of the finite element method. The parameters of the $\mathrm{MoS}_{2}$ used for the simulation are as follows: Poisson ratio 0.32 [54], Young's modulus $330 \mathrm{GPa}$ [55], mass density $4,800 \mathrm{~kg} / \mathrm{m}^{3}$, and relative permittivity 3.5 [56]. Here, the piezoelectric constant and elastic constant of the $\mathrm{MoS}_{2}$ with different widths are obtained from a DFT calculation as implemented in the ATK package. The generalized gradient approximation (GGA) using the Perdew-Burke-Ernzerhof (PBE) functional and the revised PBE functional with doublezeta polarization (DZP) orbital basis sets was used. The cut-off energy is set to $250 \mathrm{Ry}$. The tolerance of the stress error, force, and energy for structural optimization are $0.1 \mathrm{GPa}, 10^{-2} \mathrm{eV} / \AA$, and $10^{-5} \mathrm{eV}$, respectively. A density of k-mesh points of $1 \times 1 \times 7$ was used for the electronic self-consistent calculation of the nanoribbon. The piezoelectric constant of the $\mathrm{MoS}_{2}$ is calculated assuming homogeneous strains (the step of the strain is 0.01). The model sizes were limited to one lattice constant along the polar axis of the nanoribbon with periodic boundary conditions applied. The vacuum layer in the lateral and out-of-plane direction of the nanoribbon is set to $20 \AA$ to ensure non-interaction between two mirror atomic layers.

The experimental observation gives $0.5 \mathrm{C} / \mathrm{m}^{2}$ and $52 \mathrm{GPa}$ for the piezoelectric [57] and elastic constants [58] of the bulk $\mathrm{MoS}_{2}$, which is consistent with our DFT calculation (shown in Table 1). In addition, the piezoelectric constant of the nanoribbon is almost one magnitude larger than that of bulk $\mathrm{MoS}_{2}$, and the piezoelectric constant tends to decay exponentially with the nanoribbon width. Such sizedependent piezoelectricity has also been reported in $\mathrm{GaN}$ and $\mathrm{ZnO}$ nanowires [59]. This suggests that the output piezoelectric potential can be further improved by reducing the size of the materials. For this purpose, we calculated the width-dependent output voltage using the finite element simulation. As shown in Figure 4B, the peak output voltage of $3.5 \mathrm{~V}$ can be obtained when the deflection of the nanoribbon is $0.5 \mathrm{~nm}$. In addition, the output voltage can still reach around $0.5 \mathrm{~V}$, even when the piezoelectric constants approach the bulk value when the width of the nanoribbon is increased. It is still superior to the maximum output voltage that can be obtained in nanowires via the direct heating method.

From the perspective of the experiment, this result is comparable with the previous experimental observation $(0.19 \mathrm{~V}$ voltage and $28 \mathrm{pA}$ currents with $0.64 \%$ strain) for a single-layer $\mathrm{MoS}_{2}$ nanoribbon with a length of $10 \mu \mathrm{m}$ and a width of $5 \mu \mathrm{m}$. As mentioned before, the DFT calculation suggests that the piezoelectric constant of a small-size nanoribbon has a giant size effect. Therefore, an almost one order of magnitude enhancement in the output voltage has been achieved in this work. Correspondingly, a larger current can be obtained when the $\mathrm{MoS}_{2}$ nanoribbon is connected to the load. This demonstrates the great potential of nanoscale piezoelectric materials in harvesting thermal energy. In addition, it has been experimentally demonstrated that the Curie temperature of $\mathrm{MoS}_{2}$ can be effectively boosted by up to $395 \mathrm{~K}$ via a phase-transition strategy [60]. This proves the 
feasibility of the hybrid system above room temperature. Other 2D piezoelectric materials with high Curie temperatures can also serve as choices for the piezoelectric sensor of this system [61]. For structural fabrication, the concentric cross-shaped CNT network seems very complex, but similar structures have been experimentally synthesized $[62,63]$. The major difficulty is that the hybrid system is not easy to fabricate. However, the state-of-the-art practice of cutting, folding, bending, and twisting objects into versatile shapes, called nano-kirigami, has recently been identified as a feasible method for manufacturing 3D nanostructures [64]. For this reason, we believe that the hybrid structure proposed in this work will not be difficult to realize in the future.

\section{CONCLUSION}

In conclusion, we have proposed a promising mechanism for converting heat energy into electrical energy by connecting the thermophoresis effect with the piezoelectric effect. We show that the thermophoretic motion of the CNT can effectively trigger the periodic vibration of $2 \mathrm{D}$ piezoelectric materials, and the vibrational amplitude can be quantitatively controlled by the temperature difference. Moreover, the thermophoresiseffect-induced vibration of piezoelectric materials has a lower vibrational frequency than thermally induced lattice vibrations, and the size-dependent piezoelectric constant provides another way to further improve the energy conversion efficiency for this mechanism. All these features ensure a relatively high-energy conversion. This may open up a

\section{REFERENCES}

1. Gu X, Wei Y, Yin X, Li B, Yang R. Colloquium: Phononic thermal Properties of Two-Dimensional Materials. Rev Mod Phys (2018) 90:041002. doi:10.1103/ revmodphys.90.041002

2. Xiong S, Sääskilahti K, Kosevich YA, Han H, Donadio D, Volz S. Blocking Phonon Transport by Structural Resonances in alloy-based Nanophononic Metamaterials Leads to Ultralow thermal Conductivity. Phys Rev Lett (2016) 117:025503. doi:10.1103/PhysRevLett.117.025503

3. Zeng Y-J, Wu D, Cao X-H, Feng Y-X, Tang L-M, Chen K-Q. Significantly Enhanced Thermoelectric Performance of Molecular Junctions by the Twist Angle Dependent Phonon Interference Effect. J Mater Chem A (2020) 8(23): 11884-91. doi:10.1039/d0ta02423f

4. Zhang X-R, Peng X-F, Tan S-H, Long M-Q. Ballistic Electrical-thermal Transport Properties and Their Applications in Graphene-NanoribbonStacked Heterojunctions. Physica E: Low-dimensional Syst Nanostructures (2021) 136:115025. doi:10.1016/j.physe.2021.115025

5. Chen X-K, Zeng Y-J, Chen K-Q. Thermal Transport in Two-Dimensional Heterostructures. Front Mater (2020) 427:578791. doi:10.3389/fmats.2020. 578791

6. Jia P-Z, Zeng Y-J, Wu D, Pan H, Cao X-H, Zhou W-X, et al. Excellent thermoelectric performance induced by interface effect in MoS2/MoSe2 van der Waals heterostructure. J Phys Condensed Matter (2019) 32:055302. doi:10. 1088/1361-648x/ab4cab

7. Chen X-K, Hu X-Y, Jia P, Xie Z-X, Liu J. Tunable Anisotropic thermal Transport in Porous Carbon Foams: The Role of Phonon Coupling. Int J Mech Sci (2021) 206:106576. doi:10.1016/j.ijmecsci.2021.106576 new approach for developing thermally induced nanodevices for actuation and energy conversion.

\section{DATA AVAILABILITY STATEMENT}

The original contributions presented in the study are included in the article/Supplementary Material, further inquiries can be directed to the corresponding author.

\section{AUTHOR CONTRIBUTIONS}

K-QC and Y-JZ conceived this work. Y-JZ, Y-YL, HP, and Z-KD performed the numerical simulations. W-XZ, L-MT, and BL reviewed and discussed the manuscript. All authors discussed the results and contributed to the final manuscript.

\section{FUNDING}

This work was supported by the National Natural Science Foundation of China (No. 11974106).

\section{SUPPLEMENTARY MATERIAL}

The Supplementary Material for this article can be found online at: https://www.frontiersin.org/articles/10.3389/fphy.2022.823284/ full\#supplementary-material

8. Zeng Y-J, Feng Y-X, Tang L-M, Chen K-Q. Effect of Out-Of-Plane Strain on the Phonon Structures and Anharmonicity of Twisted Multilayer Graphene. Appl Phys Lett (2021) 118(18):183103. doi:10.1063/5.0047539

9. Pei Y, Shi X, LaLonde A, Wang H, Chen L, Snyder GJ. Convergence of Electronic Bands for High Performance Bulk Thermoelectrics. Nature (2011) 473(7345):66-9. doi:10.1038/nature09996

10. Pei Y, Wang H, Snyder GJ. Band Engineering of Thermoelectric Materials. $A d v$ Mater (2012) 24(46):6125-35. doi:10.1002/adma.201202919

11. Yang K, Perfetto E, Kurth S, Stefanucci G, D'Agosta R. Density Functional Theory of the Seebeck Coefficient in the Coulomb Blockade Regime. Phys Rev $B$ (2016) 94:081410. doi:10.1103/physrevb.94.081410

12. Yang J-H, Yuan Q, Deng H, Wei S-H, Yakobson BI. Earth-Abundant and Nontoxic SiX (X = S, Se) Monolayers as Highly Efficient Thermoelectric Materials. J Phys Chem C (2017) 121(1):123-8. doi:10.1021/acs.jpcc.6b10163

13. Wu D, Cao X-H, Jia P-Z, Zeng Y-J, Feng Y-X, Tang L-M, et al. Excellent Thermoelectric Performance in Weak-Coupling Molecular Junctions with Electrode Doping and Electrochemical Gating. Sci China Phys Mech Astron (2020) 63(7):276811. doi:10.1007/s11433-019-1528-y

14. Liu Q, Li J-J, Wu D, Deng X-Q, Zhang Z-H, Fan Z-Q, et al. Gate-controlled Reversible Rectifying Behavior Investigated in a Two-Dimensional MoS 2 Diode. Phys Rev B (2021) 104:045412. doi:10.1103/physrevb.104.045412

15. Wang J, Wu D, Huang L, Cao X-H, Ding Z-K, Zeng Y-J, et al. Thermoelectric Performance Enhanced by Destructive Quantum Interference in Nanoporous Carbon Nanotube Based Junctions. Phys Status Solidi RRL (2021) 15:2100400. doi:10.1002/pssr.202100400

16. Fan Z-Q, Jiang X-W, Chen J, Luo J-W. Improving Performances of In-Plane Transition-Metal Dichalcogenide Schottky Barrier Field-Effect Transistors. ACS Appl Mater Inter (2018) 10(22):19271-7. doi:10.1021/acsami.8b04860 
17. Fan Z-Q, Zhang Z-H, Yang S-Y. High-performance $5.1 \mathrm{Nm}$ In-Plane Janus WSeTe Schottky Barrier Field Effect Transistors. Nanoscale (2020) 12(42): 21750-6. doi:10.1039/d0nr05269h

18. Narducci D. Do we Really Need High Thermoelectric Figures of merit? A Critical Appraisal to the Power Conversion Efficiency of Thermoelectric Materials. Appl Phys Lett (2011) 99(10):102104. doi:10.1063/1.3634018

19. Zeng YJ, Wu D, Cao XH, Zhou WX, Tang LM, Chen KQ. Nanoscale Organic Thermoelectric Materials: Measurement, Theoretical Models, and Optimization Strategies. Adv Funct Mater (2020) 30(8):1903873. doi:10.1002/adfm.201903873

20. Zeng Y-J, Liu Y-Y, Zhou W-X, Chen K-Q. Nanoscale thermal Transport: Theoretical Method and Application. Chin Phys B (2018) 27:036304. doi:10. 1088/1674-1056/27/3/036304

21. Wang ZL, Song J. Piezoelectric Nanogenerators Based on Zinc Oxide Nanowire Arrays. Science (2006) 312(5771):242-6. doi:10.1126/science. 1124005

22. Yang R, Qin Y, Dai L, Wang ZL. Power Generation with Laterally Packaged Piezoelectric fine Wires. Nat Nanotech (2009) 4(1):34-9. doi:10.1038/nnano. 2008.314

23. Goktas N, Wilson P, Ghukasyan A, Wagner D, McNamee S, LaPierre R. Nanowires for Energy: A Review. Appl Phys Rev (2018) 5:041305. doi:10.1063/ 1.5054842

24. Matloub R, Hadad M, Mazzalai A, Chidambaram N, Moulard G, Sandu CS, et al. Piezoelectric Al1-xScxN Thin Films: A Semiconductor Compatible Solution for Mechanical Energy Harvesting and Sensors. Appl Phys Lett (2013) 102(15):152903. doi:10.1063/1.4800231

25. You Y-M, Liao W-Q, Zhao D, Ye H-Y, Zhang Y, Zhou Q, et al. An OrganicInorganic Perovskite Ferroelectric with Large Piezoelectric Response. Science (2017) 357(6348):306-9. doi:10.1126/science.aai8535

26. Sebald G, Guyomar D, Agbossou A. On Thermoelectric and Pyroelectric Energy Harvesting. Smart Mater Struct (2009) 18(12):125006. doi:10.1088/ 0964-1726/18/12/125006

27. Wu W, Wang L, Li Y, Zhang F, Lin L, Niu S, et al. Piezoelectricity of SingleAtomic-Layer MoS2 for Energy Conversion and Piezotronics. Nature (2014) 514(7523):470-4. doi:10.1038/nature13792

28. Ikuno T, Honda S-i., Yasuda T, Oura K, Katayama M, Lee JG, et al. Thermally Driven Nanomechanical Deflection of Hybrid Nanowires. Appl Phys Lett (2005) 87(21):213104. doi:10.1063/1.2133919

29. Liu Y-Y, Li B-L, Zhou W-X, Chen K-Q. Triggering Piezoelectricity Directly by Heat to Produce Alternating Electric Voltage. Appl Phys Lett (2016) 109(11): 113107. doi:10.1063/1.4962904

30. López-Suárez M, Abadal G, Gammaitoni L, Rurali R. Noise Energy Harvesting in Buckled BN Nanoribbons from Molecular Dynamics. Nano Energy (2015) 15:329-34. doi:10.1016/j.nanoen.2015.04.021

31. Schoen PAE, Walther JH, Arcidiacono S, Poulikakos D, Koumoutsakos P. Nanoparticle Traffic on Helical Tracks: Thermophoretic Mass Transport through Carbon Nanotubes. Nano Lett (2006) 6(9):1910-7. doi:10.1021/ nl060982r

32. Schoen PAE, Walther JH, Poulikakos D, Koumoutsakos P. Phonon Assisted Thermophoretic Motion of Gold Nanoparticles inside Carbon Nanotubes. Appl Phys Lett (2007) 90(25):253116. doi:10.1063/1.2748367

33. Barreiro A, Rurali R, Herna 'ndez ER, Moser J, Pichler T, Forro' L, et al. Subnanometer Motion of Cargoes Driven by thermal Gradients along Carbon Nanotubes. Science (2008) 320(5877):775-8. doi:10.1126/science.1155559

34. Guo Z, Zhang H, Li J, Leng J, Zhang Y, Chang T. An Intrinsic Energy Conversion Mechanism via Telescopic Extension and Retraction of Concentric Carbon Nanotubes. Nanoscale (2018) 10(10):4897-903. doi:10.1039/ c7nr07971k

35. Chang T, Zhang H, Guo Z, Guo X, Gao H. Nanoscale Directional Motion towards Regions of Stiffness. Phys Rev Lett (2015) 114:015504. doi:10.1103/ PhysRevLett.114.015504

36. Wang D, Chen G, Li C, Cheng M, Yang W, Wu S, et al. Thermally Induced Graphene Rotation on Hexagonal boron Nitride. Phys Rev Lett (2016) 116(12): 126101. doi:10.1103/physrevlett.116.126101

37. Takagi Y, Uda T, Ohno T. Carbon Nanotube Motors Driven by Carbon Nanotube. J Chem Phys (2008) 128(19):194704. doi:10.1063/1.2918287

38. Coluci VR, Timóteo VS, Galvão DS. Thermophoretically Driven Carbon Nanotube Oscillators. Appl Phys Lett (2009) 95(25):253103. doi:10.1063/1. 3276546
39. Guo Z, Chang T, Guo X, Gao H. Thermal-induced Edge Barriers and Forces in Interlayer Interaction of Concentric Carbon Nanotubes. Phys Rev Lett (2011) 107(10):105502. doi:10.1103/physrevlett.107.105502

40. Leng J, Guo Z, Zhang H, Chang T, Guo X, Gao H. Negative Thermophoresis in Concentric Carbon Nanotube Nanodevices. Nano Lett (2016) 16(10): 6396-402. doi:10.1021/acs.nanolett.6b02815

41. Liu Y-Y, Zhou W-X, Tang L-M, Chen K-Q. An Important Mechanism for thermal Rectification in Graded Nanowires. Appl Phys Lett (2014) 105(20): 203111. doi:10.1063/1.4902427

42. McGaughey AJ, Larkin JM. Predicting Phonon Properties from Equilibrium Molecular Dynamics Simulations. Annu Rev Heat Transfer (2014) 17:6915. doi:10.1615/annualrevheattransfer.2013006915

43. de Miguel R, Rubí JM. Negative Thermophoretic Force in the strong Coupling Regime. Phys Rev Lett (2019) 123(20):200602. doi:10.1103/physrevlett.123. 200602

44. Schelling PK, Phillpot SR, Keblinski P. Phonon Wave-Packet Dynamics at Semiconductor Interfaces by Molecular-Dynamics Simulation. Appl Phys Lett (2002) 80(14):2484-6. doi:10.1063/1.1465106

45. Liang T, Phillpot SR, Sinnott SB. Parametrization of a Reactive many-body Potential for Mo-S Systems. Phys Rev B (2009) 79(24):245110. doi:10.1103/ physrevb.79.245110

46. Stuart SJ, Tutein AB, Harrison JA. A Reactive Potential for Hydrocarbons with Intermolecular Interactions. J Chem Phys (2000) 112(14):6472-86. doi:10. $1063 / 1.481208$

47. Ding Z, Pei Q-X, Jiang J-W, Huang W, Zhang Y-W. Interfacial thermal Conductance in graphene/MoS2 Heterostructures. Carbon (2016) 96:888-96. doi:10.1016/j.carbon.2015.10.046

48. Zhou Y, Liu W, Huang X, Zhang A, Zhang Y, Wang ZL. Theoretical Study on Two-Dimensional MoS2 Piezoelectric Nanogenerators. Nano Res (2016) 9(3): 800-7. doi:10.1007/s12274-015-0959-8

49. Begtrup GE, Ray KG, Kessler BM, Yuzvinsky TD, Garcia H, Zettl A. Probing Nanoscale Solids at thermal Extremes. Phys Rev Lett (2007) 99(15):155901. doi:10.1103/physrevlett.99.155901

50. Kong LT. Phonon Dispersion Measured Directly from Molecular Dynamics Simulations. Comp Phys Commun (2011) 182(10):2201-7. doi:10.1016/j.cpc. 2011.04.019

51. Dorado B, Bottin F, Bouchet J. Phonon Spectra of Plutonium at High Temperatures. Phys Rev B (2017) 95(10):104303. doi:10.1103/physrevb.95.104303

52. Qian X, Yang R. Temperature Effect on the Phonon Dispersion Stability of Zirconium by Machine Learning Driven Atomistic Simulations. Phys Rev B (2018) 98(22):224108. doi:10.1103/physrevb.98.224108

53. Zhou WX, Cheng Y, Chen KQ, Xie G, Wang T, Zhang G. Thermal Conductivity of Amorphous Materials. Adv Funct Mater (2020) 30(8): 1903829. doi:10.1002/adfm.201903829

54. Singer IL, Bolster RN, Wegand J, Fayeulle S, Stupp BC. Hertzian Stress Contribution to Low Friction Behavior of Thin MoS2coatings. Appl Phys Lett (1990) 57(10):995-7. doi:10.1063/1.104276

55. Li T. Ideal Strength and Phonon Instability in Single-Layer MoS2. Phys Rev B (2012) 85(23):235407. doi:10.1103/physrevb.85.235407

56. Lee G-H, Yu Y-J, Cui X, Petrone N, Lee C-H, Choi MS, et al. Flexible and Transparent MoS2 Field-Effect Transistors on Hexagonal boron Nitride-Graphene Heterostructures. ACS nano (2013) 7(9):7931-6. doi:10.1021/nn402954e

57. Zhu H, Wang Y, Xiao J, Liu M, Xiong S, Wong ZJ, et al. Observation of Piezoelectricity in Free-Standing Monolayer MoS2. Nat Nanotech (2015) 10(2):151-5. doi:10.1038/nnano.2014.309

58. Feldman JL. Elastic Constants of $2 \mathrm{H}-\mathrm{MoS} 2$ and $2 \mathrm{H}-\mathrm{NbSe} 2$ Extracted from Measured Dispersion Curves and Linear Compressibilities. J Phys Chem Sol (1976) 37(12):1141-4. doi:10.1016/0022-3697(76)90143-8

59. Agrawal R, Espinosa HD. Giant Piezoelectric Size Effects in Zinc Oxide and Gallium Nitride Nanowires. A First Principles Investigation. Nano Lett (2011) 11(2):786-90. doi:10.1021/nl104004d

60. Cai L, He J, Liu Q, Yao T, Chen L, Yan W, et al. Vacancy-induced Ferromagnetism of MoS2 Nanosheets. J Am Chem Soc (2015) 137(7): 2622-7. doi:10.1021/ja5120908

61. Pan L, Wen H, Huang L, Chen L, Deng H-X, Xia J-B, et al. Two-dimensional XSe2 $(\mathrm{X}=\mathrm{Mn}, \mathrm{V})$ Based Magnetic Tunneling Junctions with High Curie Temperature*. Chin Phys. B (2019) 28(10):107504. doi:10.1088/1674-1056/ ab3e45 
62. Romo-Herrera JM, Terrones M, Terrones H, Dag S, Meunier V. Covalent 2D and 3D Networks from 1D Nanostructures: Designing New Materials. Nano Lett (2007) 7(3):570-6. doi:10.1021/nl0622202

63. Ma L, Peng J, Wu C, He L, Ni Y. Sphere-To-Tube Transition toward Nanotube Formation: A Universal Route by Inverse Plateau-Rayleigh Instability. ACS nano (2017) 11(3):2928-33. doi:10.1021/acsnano.6b08248

64. Chen S, Liu Z, Du H, Tang C, Ji CY, Quan B, et al. Electromechanically Reconfigurable Optical Nano-Kirigami. Nat Commun (2021) 12(1):1299-8. doi:10.1038/s41467-021-21565-x

Conflict of Interest: The authors declare that the research was conducted in the absence of any commercial or financial relationships that could be construed as a potential conflict of interest.
Publisher's Note: All claims expressed in this article are solely those of the authors and do not necessarily represent those of their affiliated organizations, or those of the publisher, the editors, and the reviewers. Any product that may be evaluated in this article, or claim that may be made by its manufacturer, is not guaranteed or endorsed by the publisher.

Copyright ( 2022 Zeng, Liu, Pan, Ding, Zhou, Tang, Li and Chen. This is an openaccess article distributed under the terms of the Creative Commons Attribution License (CC BY). The use, distribution or reproduction in other forums is permitted, provided the original author(s) and the copyright owner(s) are credited and that the original publication in this journal is cited, in accordance with accepted academic practice. No use, distribution or reproduction is permitted which does not comply with these terms. 